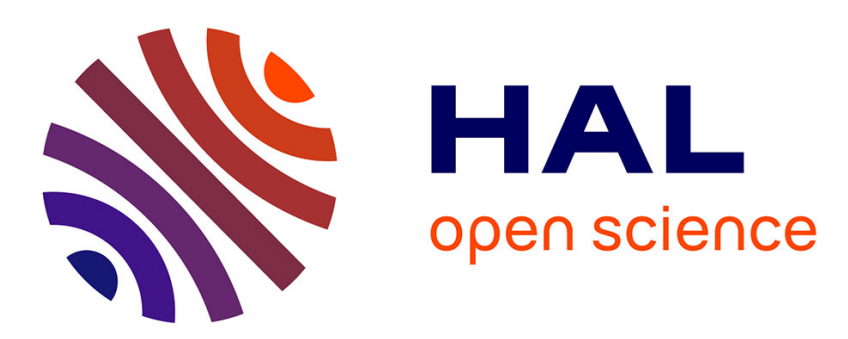

\title{
Exact Distribution and High-dimensional Asymptotics for Improperness Test of Complex Signals
}

Florent Chatelain, Nicolas Le Bihan

\section{To cite this version:}

Florent Chatelain, Nicolas Le Bihan. Exact Distribution and High-dimensional Asymptotics for Improperness Test of Complex Signals. ICASSP 2019 - IEEE International Conference on Acoustics, Speech and Signal Processing, May 2019, Brighton, United Kingdom. pp.8509-8513, 10.1109/ICASSP.2019.8683518 . hal-02148428

\section{HAL Id: hal-02148428 \\ https://hal.science/hal-02148428}

Submitted on 5 Jun 2019

HAL is a multi-disciplinary open access archive for the deposit and dissemination of scientific research documents, whether they are published or not. The documents may come from teaching and research institutions in France or abroad, or from public or private research centers.
L'archive ouverte pluridisciplinaire HAL, est destinée au dépôt et à la diffusion de documents scientifiques de niveau recherche, publiés ou non, émanant des établissements d'enseignement et de recherche français ou étrangers, des laboratoires publics ou privés. 


\title{
EXACT DISTRIBUTION AND HIGH-DIMENSIONAL ASYMPTOTICS FOR IMPROPERNESS TEST OF COMPLEX SIGNALS
}

\author{
Florent Chatelain and Nicolas Le Bihan \\ Univ. Grenoble Alpes, CNRS, Grenoble INP ${ }^{\dagger}$, GIPSA-Lab, 38000 Grenoble, France. \\ ${ }^{\dagger}$ : Institute of Engineering Univ. Grenoble Alpes
}

\begin{abstract}
Improperness testing for complex-valued vectors and processes has been of interest lately due to the potential applications of complex-valued time series analysis in several research areas. This paper provides exact distribution characterization of the GLRT (Generalized Likelihood Ratio Test) statistics for Gaussian complex-valued signals under the null hypothesis of properness. This distribution is a special case of the Wilks's lambda distribution, as are the distributions of the GLRT statistics in multivariate analysis of variance (MANOVA) procedures. In the high dimensional setting, i.e. when the size of the vectors grows at the same rate as the number of samples, a closed form expression is obtained for the asymptotic distribution of the GLRT statistics. This is, to our knowledge, the first exact characterization for the GLRT-based improperness testing.
\end{abstract}

Index Terms - Complex signals, improperness, GLRT, high-dimensional statistics, Wilks's Lambda distribution

\section{INTRODUCTION}

Complex-valued time series and vectors have attracted attention lately for their ability to model signals from a broad range of applications including digital communications [1], seismology [2], oceanography [3] or gravitational-waves physics [4] to name just a few. Among the specific features of complex datasets, the notion of properness (or second order circularity [5]), in the Gaussian case, is of major importance as it relates invariance of the probability density function to the correlation coefficients of complex vectors. The consequence of properness/circularity on complex random vectors and signals statistics was studied at large extent in [6,7]. Testing for improperness of complex vectors/signals was investigated by several authors $[8,9]$ in the signal processing community. However, it was indeed considered a long time ago by statisticians [10]. In the signal processing literature, authors have mainly used the augmented complex representation - a twice bigger vector made of the concatenation of the complex vector and its conjugate - while an equivalent real-valued representation using real and imaginary parts was preferably used by statisticians. In the sequel, we will make use of this latter representation to study the Generalized Likelihood Ratio Test (GLRT). In the scalar case, the notion of proper/improper random variable can be phrased as follows: A complex random variable is called proper if it is uncorrelated with its complex conjugate. Note that properness is a less general notion than circularity which qualifies a complex random variable whose pdf is invariant by rotation in the complex plane. In the Gaussian setting where the variable distribution depends only on second-order statistics, these two properties become equivalent. In terms of real and imaginary parts of the complex variable, properness means that both real and imaginary have the same variance and that their cross-covariance vanishes. The concept of properness extends to complex-valued vectors in a straight manner (see $[6,7]$ ).

We consider $N$-dimensional complex-valued centered random vectors $\mathbf{z}=\mathbf{u}+\boldsymbol{i} \mathbf{v}$, i.e. $\mathbf{u}$ and $\mathbf{v}$ are $N$-dimensional real vectors with zero mean. In short, we have $\mathbf{z} \in \mathbb{C}^{N}$, $\mathbf{u} \in \mathbb{R}^{N}$ and $\mathbf{v} \in \mathbb{R}^{N}$. The real vector representation of $\mathbf{z} \in \mathbb{C}^{N}$ consists in using $\mathbf{x}=\left[\mathbf{u}^{T}, \mathbf{v}^{T}\right]^{T} \in \mathbb{R}^{2 N}$. The second order statistics of $\mathbf{z} \in \mathbb{C}^{N}$ are thus contained in the real-valued covariance matrix $\mathbf{C} \in \mathbb{R}^{2 N \times 2 N}$ of $\mathbf{x}$ given by:

$$
\mathbf{C}=\left(\begin{array}{ll}
\mathbf{C}_{\mathrm{uu}} & \mathbf{C}_{\mathbf{u v}} \\
\mathbf{C}_{\mathbf{v u}} & \mathbf{C}_{\mathbf{v v}}
\end{array}\right)
$$

where $\mathbf{C}_{\mathrm{ab}} \in \mathbb{R}^{N \times N}$ denotes the real-valued (cross)covariance matrix between real vectors $\mathbf{a}$ and $\mathbf{b}$, with $\mathbf{C}_{\mathbf{b a}}=\mathbf{C}_{\mathbf{a b}}^{T}$.

A complex-valued Gaussian vector is called proper iff the following two conditions hold:

$$
\mathbf{C}_{\mathbf{u u}}=\mathbf{C}_{\mathbf{v v}} \quad \text { and } \quad \mathbf{C}_{\mathbf{u v}}^{T}=-\mathbf{C}_{\mathbf{u v}}
$$

If these conditions are not fulfilled, then $\mathbf{z}$ is called improper.

Testing for improperness of a complex Gaussian vector was studied in the seminal work of Andersson [10] where authors used the $2 N$-dimensional real-valued representation of complex $N$-dimensional vectors. They derived the maximal invariant statistics for complex vectors and characterized the joint distribution of those statistics together with using them for improperness testing. In [8, 1], authors proposed a GLRT test based on the augmented complex representation, and highlighted its connection with canonical correlation coefficients. Results showing the equivalence of the maximal in- 
variant statistics approach (derived from the $2 N$-dimensional real-valued representation) with the canonical correlation coefficients derived from the complex structure were obtained in [9], together with a numerical study of the GLRT Barlett asymptotic distribution (in the large sample size case with small or fixed dimension of the complex vector $\mathbf{z}$ ).

The original contribution of the presented work is twofold. It consists 1) in the identification of the exact distribution of GLRT statistics under the null hypothesis of properness, which reduces to a special case of Wilks's lambda distribution, and 2) in the derivation of the asymptotic distribution for the GLRT statistics in the high dimensional case (vector and sample sizes growing at the same rate).

\section{TESTING FOR IMPROPERNESS}

\subsection{Testing problem}

In several applications, it is common use to model the signal/vector of interest, denoted $\mathbf{z}$, as being improper and corrupted by proper noise. Consequently, statistical tests have been proposed to investigate the properness/improperness of a signal given a sample, from which one will decide:

$$
\begin{cases}H_{0}: & \mathbf{z} \text { is proper if condition (2) holds } \\ H_{1}: & \mathbf{z} \text { is improper otherwise }\end{cases}
$$

\subsection{Invariant parameters}

Let $\mathcal{G}$ be the set of nonsingular matrices $\mathbf{G} \in \mathbb{R}^{2 N \times 2 N}$ s.t.

$$
\mathbf{G}=\left(\begin{array}{cc}
\mathbf{G}_{1} & -\mathbf{G}_{2} \\
\mathbf{G}_{2} & \mathbf{G}_{1}
\end{array}\right)
$$

where $\mathbf{G}_{1}, \mathbf{G}_{2} \in \mathbb{R}^{N \times N}$. Let $\mathcal{S}$ be the set of all $2 N \times 2 N$ real symmetric definite positive matrices. According to the test formulation (3) and condition (2), the null hypothesis $H_{0}$ is equivalent to $\mathbf{C} \in \mathcal{T}=\mathcal{S} \cap \mathcal{G}$.

As explained in [10], $\mathcal{G}$ is a group (isomorphic to the group $G L_{N}(\mathbb{C})$ of nonsingular $N \times N$ complex matrices under the mapping $\mathbf{G} \leftrightarrow \mathbf{G}_{1}+\boldsymbol{i} \mathbf{G}_{2}$ ). Moreover $\mathcal{G}$ acts transitively on $\mathcal{T}$ under the action $(\mathbf{G}, \mathbf{T}) \in \mathcal{G} \times \mathcal{T} \mapsto \mathbf{G T G}^{T} \in$ $\mathcal{T}$. Thus, a parametric characterization of $H_{0}$ should be invariant to this group action: the value of the parameters to be tested should be the same for $\mathbf{C}$ and $\mathbf{G} \mathbf{C G}^{T}$ for any $\mathbf{G} \in \mathcal{G}$. We introduce the following decomposition $\mathbf{C}=\dot{\mathbf{C}}+\ddot{\mathbf{C}}$ for any $\mathbf{C} \in \mathcal{S}$ where

$$
\begin{aligned}
\dot{\mathbf{C}} & =\frac{1}{2}\left(\begin{array}{ll}
\mathbf{C}_{\mathbf{u u}}+\mathbf{C}_{\mathbf{v v}} & \mathbf{C}_{\mathbf{u v}}-\mathbf{C}_{\mathbf{v u}} \\
\mathbf{C}_{\mathbf{v u}}-\mathbf{C}_{\mathbf{u v}} & \mathbf{C}_{\mathbf{u u}}+\mathbf{C}_{\mathbf{v v}}
\end{array}\right) \in \mathcal{G}, \\
\ddot{\mathbf{C}} & =\frac{1}{2}\left(\begin{array}{ll}
\mathbf{C}_{\mathbf{u u}}-\mathbf{C}_{\mathbf{v v}} & \mathbf{C}_{\mathbf{u v}}+\mathbf{C}_{\mathbf{v u}} \\
\mathbf{C}_{\mathbf{u v}}+\mathbf{C}_{\mathbf{v u}} & \mathbf{C}_{\mathbf{v v}}-\mathbf{C}_{\mathbf{u u}}
\end{array}\right) .
\end{aligned}
$$

Lemma 2.1. Any matrix $\mathbf{C} \in \mathcal{S}$ can be written as:

$$
\mathbf{C}=\mathbf{G}\left(\begin{array}{cc}
\mathbf{I}_{N}+\mathbf{D}_{\lambda} & 0 \\
0 & \mathbf{I}_{N}-\mathbf{D}_{\lambda}
\end{array}\right) \mathbf{G}^{T},
$$

where $\mathbf{G} \in \mathcal{G}, \mathbf{I}_{N}$ is the $N \times N$ identity matrix and $\mathbf{D}_{\lambda}=$ $\operatorname{diag}\left(\lambda_{1}, \ldots, \lambda_{N}\right)$ is an $N \times N$ diagonal matrix. The diagonal entries of $\mathbf{D}_{\lambda}$ denoted as $\lambda_{i}$ for $1 \leq i \leq N$, are the nonnegative eigenvalues of the following $2 N \times 2 N$ real symmetric matrix

$$
\boldsymbol{\Gamma}(\mathbf{C})=\dot{\mathbf{C}}^{-\frac{1}{2}} \ddot{\mathbf{C}} \dot{\mathbf{C}}^{-\frac{1}{2}}
$$

They satisfy the following properties: 1) $\lambda_{i}$ and $-\lambda_{i}$, for $1 \leq$ $i \leq N$, form the set of eigenvalues of the $2 N \times 2 N$ matrix $\boldsymbol{\Gamma}(\mathbf{C})$, and 2) $\lambda_{i} \in[0,1]$ with, by convention, the following ordering $1 \geq \lambda_{1} \geq \ldots \geq \lambda_{N} \geq 0$.

Proof. See [10, lemma 5.1 and 5.2].

Lemma 2.1 shows that any invariant parameterization of the covariance matrix $\mathbf{C}$ for the group action of $\mathcal{G}$ depends only on the $N$ (positive) eigenvalues $1 \geq \lambda_{1} \geq \ldots \geq \lambda_{N} \geq$ 0 . Thus these eigenvalues are termed as maximal invariant parameters [11, Chapter 6]. Moreover under the null hypothesis $H_{0}$, it comes that $\lambda_{1}=\ldots=\lambda_{N}=0$ as $\ddot{\mathbf{C}}$ reduces to the zero matrix according to (2). Within the invariant parameterization, the testing problem in (3) becomes

$$
\begin{cases}H_{0}: & \lambda_{i}=0, \text { for } 1 \leq i \leq N \\ H_{1}: & \lambda_{i} \geq 0, \text { for } 1 \leq i \leq N\end{cases}
$$

Note that the invariance property ensures that the test does not depend on the (common) representation basis of the real and imaginary parts of $\mathbf{z}$, i.e. vectors $\mathbf{u}$ and $\mathbf{v}$.

\subsection{Invariant statistics}

Consider we have a sample of size $M$, denoted $\mathbf{X}=\left\{\mathbf{x}_{m}\right\}_{m=1}^{M}$, where $\mathbf{x}_{m}=\left[\mathbf{u}_{m}^{T}, \mathbf{v}_{m}^{T}\right]^{T}$ are $2 N$-dimensional i.i.d. Gaussian real vectors with zero mean and covariance matrix $\mathbf{C}$. In the Gaussian framework, a sufficient statistics is given by the $2 N \times 2 N$ sample covariance matrix

$$
\mathbf{S}=\left(\begin{array}{ll}
\mathbf{S}_{\mathbf{u u}} & \mathbf{S}_{\mathbf{u v}} \\
\mathbf{S}_{\mathbf{v u}} & \mathbf{S}_{\mathbf{v v}}
\end{array}\right)
$$

with $\mathbf{S}_{\mathbf{a b}} \in \mathbb{R}^{N \times N}$ the real-valued sample (cross)covariance matrix of real vectors $\left\{\mathbf{a}_{m}\right\}_{m=1}^{M}$ and $\left\{\mathbf{b}_{m}\right\}_{m=1}^{M}$ such that $\mathbf{S}_{\mathbf{a b}}=\frac{1}{M} \sum_{m=1}^{M} \mathbf{a}_{m} \mathbf{b}_{m}^{T}$. We assume here that $M \geq 2 N$, thus $\mathbf{S}$ belongs to the real symmetric definite positive matrix set $\mathcal{S}$. According to previous section, since $H_{0}$ is invariant under the action of group $\mathcal{G}$, an invariant test statistics must only depend on the $N$ positive eigenvalues $l_{i}, 1 \leq i \leq N$, of $\boldsymbol{\Gamma}(\mathbf{S})=\dot{\mathbf{S}}^{-\frac{1}{2}} \ddot{\mathbf{S}}^{-\frac{1}{2}}$. These sample eigenvalues obey $1 \geq l_{1} \geq \ldots \geq l_{N} \geq 0$ according to lemma 2.1, and 
are an estimate of the true eigenvalues $\lambda_{i}$ obtained from the population covariance $\mathbf{C}$. Note that the $\lambda_{i}$ are zero under the null hypothesis $H_{0}$, and non-negative otherwise. As a consequence, the distribution of the $l_{i}$ should be stochastically greater under $H_{1}$ than under $H_{0}$. Any invariant test can be derived from this property. A key point to derive now a tractable statistical test procedure is to characterize the null distribution of these eigenvalues.

Let $\mathcal{B}_{N}\left(\frac{1}{2} n_{1}, \frac{1}{2} n_{2}\right)$ denote the $N \times N$-dimensional matrix variate beta distribution with parameters $n_{1}$ and $n_{2}$ as defined for instance in [12, definition 3.3.2, p. 110].

Proposition 2.1.1. Under $H_{0}$, the vector $\left(r_{1}, \ldots, r_{N}\right)$ of the squared sample eigenvalues $r_{n}=l_{n}^{2}$ is distributed as the eigenvalues of the matrix variate beta distribution $\mathcal{B}_{N}\left(\frac{1}{2} n_{1}, \frac{1}{2} n_{2}\right)$, with parameters $n_{1}=N+1$ and $n_{2}=$ $M-N$. Moreover, the joint pdf of $\left(r_{1}, \ldots, r_{N}\right)$ is expressed as:

$$
p\left(r_{1}, \ldots, r_{N}\right) \propto \prod_{n=1}^{N}\left(1-r_{n}\right)^{(M-2 N-1) / 2} \prod_{k<n}^{N}\left(r_{k}-r_{n}\right)
$$

where $1 \geq r_{1} \geq \ldots \geq r_{N} \geq 0$.

Proof. As shown in [10, pp. 39-41], the sample eigenvalue vector $\left(l_{1}, \ldots, l_{N}\right)$ is characterized by the following probability density function (pdf):

$p\left(l_{1}, \ldots, l_{N}\right) \propto \prod_{n=1}^{N}\left(2 l_{n}\right)\left(1-l_{n}^{2}\right)^{(M-2 N-1) / 2} \prod_{k<n}^{N}\left(l_{k}^{2}-l_{n}^{2}\right)$.

A simple change of variables yields the pdf of $\left(r_{1}, \ldots, r_{N}\right)$ given in (5). Moreover, according to [12, Theorem 3.3.4, p. 112], (5) is the pdf of the eigenvalues of the matrix variate beta distribution $\mathcal{B}_{N}\left(\frac{N+1}{2}, \frac{M-N}{2}\right)$, which concludes the proof.

\section{GENERALIZED LIKELIHOOD RATIO TEST}

\subsection{Expression of the GLRT statistic}

A very classical procedure to test for improperness is obtained from the Generalized Likelihood Ratio Test (GLRT) statistic defined as:

$$
T \propto \frac{\sup _{\mathbf{C} \text { s.t. } H_{0}} p(\mathbf{X} ; \mathbf{C})}{\sup _{\mathbf{C} \text { s.t. } H_{1}} p(\mathbf{X} ; \mathbf{C})}
$$

where $p(\mathbf{X} ; \mathbf{C})$ is the multivariate normal pdf of the sample $\mathrm{X}$ composed of $M$ i.i.d. $2 N$-dimension real Gaussian vectors with zero mean and covariance matrix $\mathbf{C}$. Under $H_{1}, \mathbf{C} \in \mathcal{S}$ is a symmetric definite positive matrix. It is well known that its maximum likelihood (ML) estimate is the sample covariance $\mathbf{S}$. Under $H_{0}$, it comes that $\mathbf{C}=\dot{\mathbf{C}}$ as $\mathbf{C} \in \mathcal{T}$. Then the ML estimate of $\mathbf{C}$ under $H_{0}$ reduces to $\dot{\mathbf{S}}$, as shown for instance in [10]. Actually, the GLRT statistics is expressed as:

$$
\begin{aligned}
T & =|\mathbf{S}| /|\dot{\mathbf{S}}|=\left|\dot{\mathbf{S}}^{\frac{1}{2}}\left(\mathbf{I}_{2 N}+\boldsymbol{\Gamma}(\mathbf{S})\right) \dot{\mathbf{S}}^{\frac{1}{2}}\right| /|\dot{\mathbf{S}}|=\left|\mathbf{I}_{2 N}+\Gamma(\mathbf{S})\right| \\
& =\prod_{n=1}^{N}\left(1+l_{n}\right)\left(1-l_{n}\right)=\prod_{n=1}^{N}\left(1-r_{n}\right)
\end{aligned}
$$

where the first equality in the first line is due to the Gaussian pdf expression, the second equality comes from the decomposition $\mathbf{S}=\dot{\mathbf{S}}+\ddot{\mathbf{S}}$ and the definition of $\boldsymbol{\Gamma}(\mathbf{S})$, the first equality in the second line comes from lemma 2.1 , and where $r_{n}=l_{n}^{2}, 1 \leq n \leq N$, are the squared sample eigenvalues. As explained in the previous section, it is important to note that the GRLT is invariant: the resulting statistics given in (6) only depends on the eigenvalues of $\boldsymbol{\Gamma}(\mathbf{S})$.

\subsection{Distribution under the hypothesis $H_{0}$ of properness}

Let $\Lambda(d, m, n)$ denote the Wilks's lambda distribution, with dimension parameter $d$ and degrees of freedom parameters $m$ and $n$, as defined for instance in [13, definition 3.7.1, p. 81].

Theorem 3.1. The GLRT statistics $T$ given in (6) is distributed under $H_{0}$ as the following Wilks's lambda distribution:

$$
T \sim \Lambda(N, M-N, N+1)
$$

Moreover this statistics can be expressed under $H_{0}$ as

$$
T=\prod_{n=1}^{N} u_{n}
$$

where the $u_{n}$ are independent beta-distributed random variables such that $u_{n} \sim \mathcal{B}\left(\frac{M-N-n+1}{2}, \frac{N+1}{2}\right)$, for $1 \leq n \leq N$.

Proof. According to Prop. 2.1.1, the $r_{n}$ in (6) are distributed as the eigenvalues of the matrix variate beta distribution $\mathcal{B}_{N}\left(\frac{1}{2} n_{1}, \frac{1}{2} n_{2}\right)$ with parameters $n_{1}=N+1$ and $n_{2}=M-N$. Using the mirror symmetry property of the beta distribution, it comes that the $\left(1-r_{n}\right)$ are distributed as the eigenvalues of the random matrix $\mathbf{U} \sim \mathcal{B}_{N}\left(\frac{1}{2} n_{2}, \frac{1}{2} n_{1}\right)$. According now to [12, Theorem 3.3.3, p. 110], $\mathrm{U}$ can be decomposed as $\mathbf{U}=\boldsymbol{\Theta}^{T} \boldsymbol{\Theta}$ where $\boldsymbol{\Theta}$ is upper triangular with diagonal entries $\theta_{n n}$ that are independent and where $u_{n} \equiv \theta_{n n}^{2} \sim \mathcal{B}\left(\frac{n_{2}-n+1}{2}, \frac{n_{1}}{2}\right)$ for $1 \leq n \leq N$. This concludes the proof.

Equation (7) gives also a more efficient way to sample from the null distribution of $T$ in $O(N)$ independent draws. This does not require to generate the $2 N \times 2 N$ sample covariance matrix $\mathbf{S}$, nor to compute the eigenvalues of $\boldsymbol{\Gamma}(\mathbf{S})$. 


\subsection{High-dimensional asymptotic distribution under $H_{0}$}

The characterization given in (7) allows us to derive, under the null hypothesis $H_{0}$, an asymptotic distribution for the GLRT statistic $T$ in the high dimensional (i.e. large $N$ ) case. This yields a simple tractable closed form approximation of the considered Wilks's lambda distribution when both the dimension $N$ and the sample size $M$ are large.

Theorem 3.2. Let $T^{\prime}=-\ln T$ where $T$ is the GLRT statistic given in (6). Assume that $M, N \rightarrow \infty$ so that the ratio $M / N \rightarrow \gamma \in(2,+\infty)$. Under $H_{0}$, the following asymptotic normal distribution is obtained for $T^{\prime}$

$$
\frac{1}{s_{M}}\left(T^{\prime}-m_{M}\right) \stackrel{d}{\rightarrow} \mathcal{N}(0,1)
$$

where

$$
\begin{aligned}
m_{M} & =M\left[\ln \frac{\gamma}{\gamma-1}+\frac{\gamma-2}{\gamma} \ln \frac{\gamma-2}{\gamma-1}\right]+\frac{1}{2} \ln \frac{\gamma}{\gamma-2}, \\
s_{M}^{2} & =2\left[\ln \frac{(\gamma-1)^{2}}{\gamma(\gamma-2)}+\frac{1}{M} \frac{1}{\gamma-2}\right] .
\end{aligned}
$$

Proof. According to theorem 3.1, $T^{\prime}=\sum_{n=1}^{N} \zeta_{n}$ where the $\zeta_{n}$ are independent random variables such that $\zeta_{n}=-\ln u_{n}$ with $u_{n} \sim \mathcal{B}\left(\frac{M-N-n+1}{2}, \frac{N+1}{2}\right)$ for $1 \leq n \leq N$. Based on the centered moments of a logarithmically transformed beta-distributed variable as given in [14], it comes that $E\left[\zeta_{n}\right]=\psi\left(a_{n}+b\right)-\psi\left(a_{n}\right)$ where $\psi(\cdot)$ is the digamma function, and $\operatorname{var}\left[\zeta_{n}\right]=\psi_{1}\left(a_{n}\right)-\psi_{1}\left(a_{n}+b\right)$ where $\psi_{1}(\cdot)$ is the trigamma function. Using Taylor series expansions of the digamma and trigamma functions, straightforward computations, omitted here for the sake of brevity, yield that $E\left[T^{\prime}\right]=\sum_{n=1}^{N} E\left[\zeta_{n}\right]=m_{M}+O(1 / M)$ and $\operatorname{var}\left(T^{\prime}\right)=\sum_{n=1}^{N} \operatorname{var}\left(\zeta_{n}\right)=s_{M}^{2}+O\left(1 / M^{2}\right)$.

In order to apply Lyapunov central limit theorem $[15, \mathrm{p}$. 362] to $T^{\prime}=\sum_{n=1}^{N} \zeta_{n}$, it is sufficient to show that

$$
\frac{1}{\operatorname{var}\left(T^{\prime}\right)^{2}} \sum_{n=1}^{N} E\left[\left(\zeta_{n}-E\left[\zeta_{n}\right]\right)^{4}\right] \rightarrow 0 .
$$

The expression of the fourth order centered moment of $\zeta_{n}$ gives now that $E\left[\left(\zeta_{n}-E\left[\zeta_{n}\right]\right)^{4}\right]=O\left(1 /(M-n+2)^{2}\right)$ for $1 \leq n \leq N$. As $\operatorname{var}\left(T^{\prime}\right)=s_{M}^{2}+O\left(1 / M^{2}\right)=O(1)$, the previous Lyapunov sufficient condition holds, and

$$
Z \equiv \frac{1}{\sqrt{\operatorname{var}\left(T^{\prime}\right)}} \sum_{n=1}^{N}\left(\zeta_{n}-E\left[\zeta_{n}\right]\right) \stackrel{d}{\rightarrow} \mathcal{N}(0,1) .
$$

By noting finally that $\frac{1}{s_{M}}\left(T^{\prime}-m_{M}\right)=Z+O(1 / M)$, Slutsky's theorem allows us to conclude the proof.

\section{SIMULATION RESULTS}

Several simulations have been conducted to appreciate the accuracy of the asymptotic null distribution given in Thm. 3.2 with respect to 1) the sample size $M$ and 2) the dimension $N$, or equivalently the ratio $\gamma=\frac{M}{N}$. This approximation is also compared with the classical Bartlett one derived for Wilks's lambda distribution [13, p. 94]. This gives, when the dimension $N$ is fixed while $M$ goes to infinity, the same asymptotic distribution as obtained in [9]:

$$
-(M-N) \ln T \stackrel{d}{\rightarrow} \chi_{N(N+1)}^{2},
$$

where $\chi_{N(N+1)}^{2}$ denotes the chi-squared distribution with $N(N+1)$ degrees of freedom.

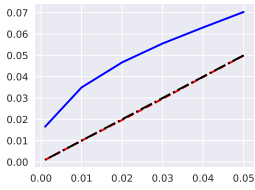

(a) $\gamma=10, M=10$

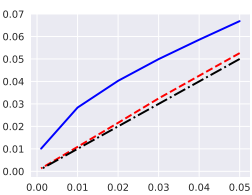

(d) $\gamma=5, M=10$

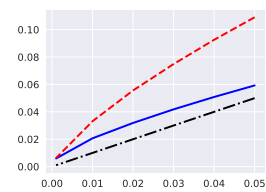

(g) $\gamma=2.5, M=10$

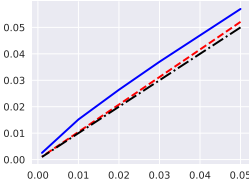

(b) $\gamma=10, M=100$

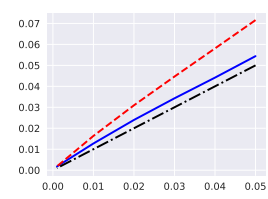

(e) $\gamma=5, M=100$

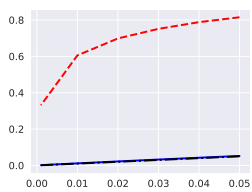

(h) $\gamma=2.5, M=100$

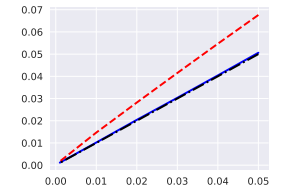

(c) $\gamma=10, M=1000$

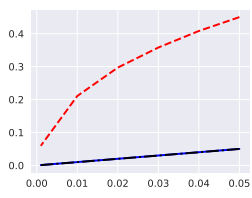

(f) $\gamma=5, M=1000$

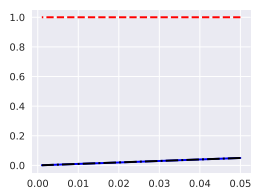

(i) $\gamma=2.5, M=1000$
Fig. 1: Comparison of asymptotic approximations for the GLRT statistics $T: \operatorname{Pr}\left(T>q_{\alpha}\right)$ under $H_{0}$ vs the nominal control level $\alpha$ in $\left[10^{-3}, 5 \times 10^{-1}\right]$ where $q_{\alpha}$ is the $1-\alpha$ quantile either for the lognormal approximation given in theorem 3.2, shown in solid blue line, or the Bartlett approximation (8), shown in dashed red line. (a)-(c) are for $\gamma=M / N=10$ and $M=10,100,1000$ respectively, (d)(f) are likewise for $\gamma=M / N=5$ and (g)-(i) for $\gamma=M / N=2.5$. The black dashdotted line represents the $y=x$ values.

Fig. 1 depicts, for different values of $M$ and $\gamma$, a probability-probability plot of the theoretical null distribution of $T$ against each one of these asymptotic approximations. A deviation from the $y=x$ line indicates a difference between the theoretical and the asymptotic distributions. This shows that as expected for high-dimensional setting (e.g., $\gamma \leq 5$ ) and/or large sample sizes (e.g., $M \geq 1000$ ), the asymptotic distribution that we derived becomes very accurate and much better than Bartlett one. 


\section{REFERENCES}

[1] P.J. Schreier and L.L. Scharf, Statistical Signal Processing of Complex-Valued Data: The Theory of Improper and Noncircular Signals, Cambridge University Press, 2010 .

[2] A. M. Sykulski, S. C. Olhede, and J. M. Lilly, "A widely linear complex autoregressive process of order one," IEEE Transactions on Signal Processing, vol. 64, no. 23, pp. 6200-6210, 2016.

[3] A. M. Sykulski, S. C. Olhede, J. M. Lilly, and J. J. Early, "Frequency-domain stochastic modeling of stationary bivariate or complex-valued signals," IEEE Transactions on Signal Processing, vol. 65, no. 12, pp. 31363151, 2017.

[4] J. Flamant, P. Chainais, E. Chassande-Mottin, F. Feng, and N. Le Bihan, "Non-parametric characterization of gravitational-wave polarizations," in $X X V$ European Signal Processing Conference (EUSIPCO), Roma, Italy, 2018.

[5] B. Picinbono, "On circularity," IEEE Trans. on Signal Processing, vol. 42, no. 12, pp. 3473-3482, 1994.

[6] P.-O. Amblard, M. Gaëta, and J.-L. Lacoume, "Statistics for complex variables and signals, Part I," Signal Processing, vol. 53, pp. 1-13, 1996.

[7] P.-O. Amblard, M. Gaëta, and J.-L. Lacoume, "Statistics for complex variables and signals, Part II," Signal Processing, vol. 53, pp. 15-25, 1996.

[8] P. J. Schreier, L. L. Scharf, and A. Hanssen, "A generalized likelihood ratio test for impropriety of complex signals," IEEE Signal Processing Letters, vol. 13, no. 7, pp. $433-436,2006$.

[9] A.T. Walden and P. Rubin-Delanchy, "On testing for improperty of complex-valued gaussian vectors," IEEE Transactions on Signal Processing, vol. 57, no. 3, pp. 21-51, 2009.

[10] S.A. Andersson and M.D. Perlman, "Two testing problems relating the real and complex multivariate normal distributions," Journal of Multivariate Analysis, vol. 15, pp. 21-51, 1975.

[11] E. L. Lehmann and Joseph P. Romano, Testing statistical hypotheses, Springer Texts in Statistics. Springer, New York, third edition, 2005.

[12] R.J. Muirhead, Aspects of Multivariate Statistical Theory, Wiley-Interscience, 2005.

[13] K. V. Mardia, J. T. Kent, and J. M. Bibby, Multivariate analysis, Academic Press London ; New York, 1979.
[14] S. Nadarajah and S. Kotz, "The beta exponential distribution," Reliability Engineering \& System Safety, vol. 91, no. 6, pp. $689-697,2006$.

[15] P. Billingsley, Probability and Measure, Wiley Series in Probability and Statistics. Wiley, 1995. 